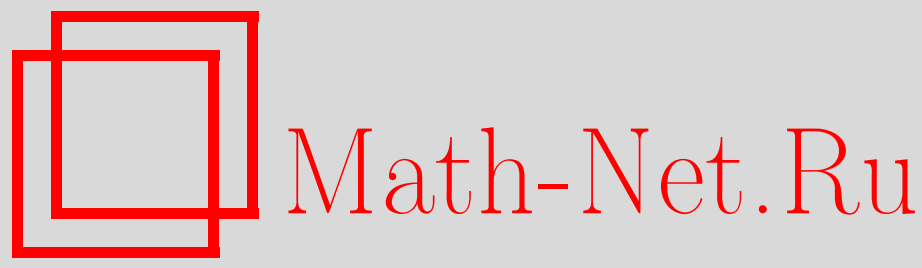

Н. А. Щучкин, Строение конечных абелевых $n$-арных групп, Дискрет. матем., 2014, том 26, выпуск 3, 144159

DOI: https://doi.org/10.4213/dm1298

Использование Общероссийского математического портала Math-Net.Ru подразумевает, что вы прочитали и согласны с пользовательским соглашением http://www . mathnet.ru/rus/agreement

Параметры загрузки:

IP : 54.210 .77 .194

26 апреля 2023 г., 17:09:58

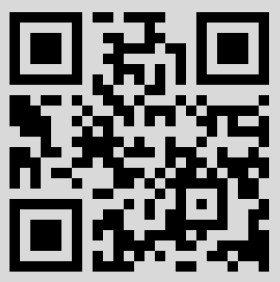




\title{
Строение конечных абелевых $n$-арных групп
}

() 2014 г. Н.А.Щучкин*

\begin{abstract}
Приведено полное описание строения конечных абелевых $n$-арных групп в виде прямого произведения примарных абелевых полуциклических $n$-арных групп с точностью до изоморфизма.
\end{abstract}

Ключевые слова: квазигруппы, $n$-арные группы, структура групп.

\section{1. Основные сведения из теории $n$-арных групп}

Алгебру $\langle G, f\rangle$ с одной $n$-арной операцией $f(n \geqslant 3)$ называют $n$-арной квазигруп$n o u ̈$, если в ней для каждого $j=1, \ldots, n$ разрешимо и имеет единственное решение уравнение

$$
f\left(a_{1}, \ldots, a_{j-1}, x_{j}, a_{j+1}, \ldots, a_{n}\right)=b
$$

при любых $a_{1}, \ldots, a_{j-1}, a_{j+1}, \ldots, a_{n}, b$ из $G$. Частным случаем понятия $n$-арной квазигруппы является обобщение понятия группы на $n$-арный случай. Именно, $n$-арная квазигруппа $\langle G, f\rangle$ называется $n$-арной группой, если в ней выполняется обобщенный закон ассоциативности

$$
f\left(f\left(a_{1}, \ldots, a_{n}\right), a_{n+1}, \ldots, a_{2 n-1}\right)=f\left(a_{1}, \ldots, a_{i}, f\left(a_{i+1}, \ldots, a_{i+n}\right), a_{i+n+1}, \ldots, a_{2 n-1}\right)
$$

для всех $i=1, \ldots, n-1$ (см. стр. 52, [1]).

Если в $n$-арной группе для любой подстановки $\sigma \in S_{n}$ верны тождества

$$
f\left(x_{1}, \ldots, x_{n}\right)=f\left(x_{\sigma(1)}, \ldots, x_{\sigma(n)}\right),
$$

то она называется абелевой. Теория $n$-арных групп существенно отличается от теории групп, однако имеется тесная связь между группами и $n$-арными группами. В [2] показано, что на любой абелевой $n$-арной группе $\langle G, f\rangle$ можно определить абелеву группу $\operatorname{red}_{c}\langle G, f\rangle$ с бинарной операцией + по правилу: $a+b=f(a, \underbrace{c, \ldots, c}_{n-3}, \bar{c}, b)$,

где $c-$ фиксированный элемент из $G$. Тогда

$$
f\left(a_{1}, \ldots, a_{n}\right)=a_{1}+\ldots+a_{n}+d
$$

где $d=f(\underbrace{c, \ldots, c}_{n})$. Элемент $c$ является нулем в группе $\operatorname{red}_{c}\langle G, f\rangle$. Верно и обратное: в любой абелевой группе $G$ для выбранного элемента $d$ существует абелева $n$-арная

* Место работы: Волгоградский государственный социально-педагогический ун-т, e-mail: nikolaj_shchuchkin@mail.ru 
группа $\langle G, f\rangle=a b l_{d} G$, где $f$ действует по правилу (1). Кроме того, $\langle G, f\rangle=a b l_{d} G$ тогда и только тогда, когда $G=\operatorname{red}_{0}\langle G, f\rangle$ и $d=f(\underbrace{0, \ldots, 0}_{n})$ (см. [2]).

Таким образом, абелевы $n$-арные группы и соответствующие им абелевы группы полиномиально эквивалентны. А поскольку у полиномиально эквивалентных алгебр совпадают решетки конгруэнций, то вопросы существования разложения в прямые произведения для них решаются тождественным образом. Ответы на эти вопросы могут отличаться лишь в плане единственности, так как за счет различного выбора констант $d$ изоморфным группам могут быть полиномиально эквивалентны не изоморфные $n$-арные группы. При этом имеется критерий изоморфизма абелевых $n$-арных групп.

Теорема 1 (Следствие 17, [3]). Изоморфизм $n$-арных груnп $\langle G, f\rangle=a b l_{d} G u$ $\left\langle G^{\prime}, f^{\prime}\right\rangle=a b l_{d^{\prime}} G^{\prime}$ существует тогда и только тогда, когда существуют такие изоморфизм $\sigma$ из группъ $G$ в группу $G^{\prime}$ и элемент $u \in G^{\prime}$, что

$$
\sigma(d)=(n-1) u+d^{\prime} .
$$

Как и в теории групп, конечную $n$-арную группу, порядок которой есть степень простого числа $p$, назовем $n$-арной $p$-группой. Если $n$-арная группа является $n$-арной $p$-группой для некоторого простого числа $p$, то она называется примарной.

Теорема 2 (Теорема 2, [4]). Любая абелева $n$-арная группа $\langle G, f\rangle$, порядок которой равен $p_{1}^{\alpha_{1}} p_{2}^{\alpha_{2}} \ldots p_{k}^{\alpha_{k}}\left(p_{i}-\right.$ простые числа), изоморфна прямому произведению

$$
\left\langle G_{1}, f_{1}\right\rangle \times\left\langle G_{2}, f_{2}\right\rangle \times \ldots \times\left\langle G_{k}, f_{k}\right\rangle
$$

$n$-арных $p_{i}$-групп $\left\langle G_{i}, f_{i}\right\rangle$ порядков $p_{i}^{\alpha_{i}}$.

Разложение в теореме 2 определено однозначно (см. Теорема 3, [4]). Наша цель заключается в том, чтобы конечную абелеву $n$-арную группу представить в виде прямого произведения простейших $n$-арных групп, каковыми являются $n$-арные группы $a b l_{l a}(a)$, где $(a)$ - циклическая группа (их еще называют абелевыми полуциклическими). Результаты, полученные ниже, дополняют сведения о строении конечных $n$-арных групп, приведенные в работах [5], [6].

\section{2. Конечные примарные абелевы $n$-арные группы.}

Согласно теореме 2 любая примарная абелева $n$-арная группа порядка $p^{m}$ изоморфна прямому произведению $k$ абелевых $n$-арных групп порядков $p^{m_{i}}$, где $m=$ $m_{1}+\ldots+m_{k}$. В отличие от общего случая, примарная абелева полуциклическая $n$-арная группа не может быть изоморфна прямому произведению нескольких $n$-арных групп меньшего порядка (Предложение 13, [7]). Именно такие неразложимые абелевы $n$-арные группы служат компонентами прямого разложения примарной абелевой $n$-арной группы.

Теорема 3 (Теорема 8, [4]). Каждая конечная абелева $n$-арная р-группа изоморфна прямому произведению абелевых полуииклических п-арных р-групп.

Например, абелева тернарная $(n=3)$ группа $a b l_{a+b} G$ порядка 16, где $G=(a)+(b)$ - прямая сумма циклических групп $(a)$ и $(b)$ порядков 8 и 2 соответственно, изоморфна прямому произведению $a b l_{a}(a) \times a b l_{b}(b)$ абелевых полуциклических $n$-арных 
групп. Действительно, $n$-арная группа $a b l_{a}(a) \times a b l_{b}(b)=a b l_{(a, b)} G$, где $G$ - внешняя прямая сумма циклических групп $(a)$ и $(b)$. Тождественный автоморфизм группы $G$ будет изоморфизмом $n$-арных групп $a b l_{a+b} G$ и $a b l_{a}(a) \times a b l_{b}(b)$.

В теории групп каждая абелева $p$-группа определяется однозначно с точностью до изоморфизма своими инвариантами $p^{m_{1}}, \ldots, p^{m_{t}}$. Для $n$-арных групп это не так. Даже абелевы полуциклические $n$-арные группы одного и того же порядка могут быть не изоморфными. Известно (см. Лемма 1, [9]), что любые две $n$-арные группы $a b l_{l_{1} a}(a)$ и $a b l_{l_{2} a}(a)$ изоморфны тогда и только тогда, когда НОД $\left(l_{1}, n-1, k\right)=$ НОД $\left(l_{2}, n-1, k\right)$, где $|(a)|=k$. Отсюда следует, что количество различных (с точностью до изоморфизма) конечных абелевых полуциклических $n$-арных групп одного и того же порядка $k$ равно количеству натуральных делителей $\tau(\mathrm{HOД}(n-$ $1, k))$ числа НОД $(n-1, k)$. Напрашивается гипотеза (по аналогии с теорией групп): прямое произведение

$$
a b l_{l_{1} a_{1}}\left(a_{1}\right) \times \ldots \times a b l_{l_{r} a_{r}}\left(a_{r}\right),
$$

абелевых полуциклических $n$-арных $p$-групп $a b l_{l_{i} a_{i}}\left(a_{i}\right)$ однозначно (с точностью до изоморфизма) определяется количеством прямых множителей в разложении (3) и изоморфными копиями множителей из (3). Однако это не так. Например, прямое произведение $a b l_{a}(a) \times a b l_{b}(b)$, где $|(a)|=8,|(b)|=2$, изоморфно прямому произведению $a b l_{a}(a) \times a b l_{0}(b)$. Действительно, $a b l_{a}(a) \times a b l_{b}(b)=a b l_{a+b} G$, $a b l_{a}(a) \times a b l_{0}(b)=a b l_{a} G$, где $G=(a)+(b)$ - прямая сумма циклических групп $(a)$ и $(b)$. Имеется автоморфизм $\sigma$ группы $G$, переводящий базис $a, b$ в базис $a+b, b$. При таком автоморфизме верно равенство $\sigma(a+b)=a$, а значит, по теореме 1 имеем искомый изоморфизм. Однако вторые множители $a b l_{b}(b)$ и $a b l_{0}(b)$ не изоморфны (по Лемме 1 из [9]).

Для нахождения условий однозначности разложения (3) введем новое определение. Множители с равными порядками в разложении (3) расположим рядом, и такие подпрямые произведения расположим в порядке убывания порядков множителей, входящих в эти подпрямые произведения. Получим разложение

$$
\prod_{i=1}^{r_{1}} a b l_{l_{i 1} a_{i 1}}\left(a_{i 1}\right) \times \prod_{i=1}^{r_{2}} a b l_{l_{i 2} a_{i 2}}\left(a_{i 2}\right) \times \ldots \times \prod_{i=1}^{r_{t}} a b l_{l_{i t} a_{i t}}\left(a_{i t}\right)
$$

и если $\left|\left(a_{i j}\right)\right|=p^{m_{j}}$ для $j=1, \ldots, t$ и каждого $i=1, \ldots, r_{j}$, то $m_{1}>m_{2}>\ldots>m_{t}$. Для разложения (4) назовем определяющим набор $D_{1}, \ldots, D_{t}$ наибольших общих делителей, заданных по правилу

$$
\left\{\begin{array}{l}
D_{1}=\text { НОД }\left(d_{1}, p^{m_{1}-m_{2}} d_{2}, \ldots, p^{m_{1}-m_{t}} d_{t}, p^{m_{1}}, n-1\right), \\
D_{2}=\text { НОД }\left(d_{1}, d_{2}, p^{m_{2}-m_{3}} d_{3}, \ldots, p^{m_{2}-m_{t}} d_{t}, p^{m_{2}}, n-1\right), \\
\cdots \\
D_{t-1}=\text { НОД }\left(d_{1}, \ldots, d_{t-1}, p^{m_{t-1}-m_{t}} d_{t}, p^{m_{t-1}}, n-1\right), \\
D_{t}=\text { НОД }\left(d_{1}, \ldots, d_{t-1}, d_{t}, p^{m_{t}}, n-1\right),
\end{array}\right.
$$

где $d_{j}=$ НОД $\left(l_{1 j}, \ldots, l_{r_{j} j}\right)$ для всех $j=1, \ldots, t$. Теперь можно доказать теорему о единственности разложения конечной абелевой $n$-арной $p$-группы в прямое произведению абелевых полуциклических $n$-арных $p$-групп.

Теорема 4. Если конечная абелева $n$-арная $p$-группа $\langle G, f\rangle$ изоморфна двум прямым произведениям абелевых полуииклических n-арных р-групп: (3) $и$

$$
a b l_{l_{1}^{\prime} b_{1}}\left(b_{1}\right) \times \ldots \times a b l_{l_{s}^{\prime} b_{s}}\left(b_{s}\right),
$$


то $r=s$, порядки $\left|\left(a_{i}\right)\right|$ совпадают с порядками $\left|\left(b_{j}\right)\right|$ при некотором упорядочении последних и определяющие наборы наибольших общих делителей для разложений (3) и (6) одинаковы.

Доказательство. Заметим, что $n$-арная группа (3) равна $a b l_{d} A$, где $A=\sum_{i=1}^{r}\left(a_{i}\right)$, $d=\sum_{i=1}^{r} l_{i} a_{i}$, а $n$-арная группа (6) равна $a b l_{d^{\prime}} B$, где $B=\sum_{i=1}^{s}\left(b_{i}\right), d^{\prime}=\sum_{i=1}^{s} l_{i}^{\prime} b_{i}$. Группы $A$ и $B$ изоморфны (это следует из изоморфизма $n$-арных групп (3) и (6) по теореме 1). В силу единственности разложения конечной абелевой $p$-группы в прямое произведение циклических $p$-групп имеем $r=s$ и $\left|\left(a_{i}\right)\right|=\left|\left(b_{j}\right)\right|$ при соответствующей нумерации последних.

Для доказательства равенства определяющих наборов наибольших общих делителей $n$-арных групп (3) и (6) упорядочим разложения (3) в виде (4) и (6) следующим образом:

$$
\prod_{i=1}^{r_{1}} a b l_{l_{i 1}^{\prime} b_{i 1}}\left(b_{i 1}\right) \times \prod_{i=1}^{r_{2}} a b l_{l_{i 2}^{\prime} b_{i 2}}\left(b_{i 2}\right) \times \ldots \times \prod_{i=1}^{r_{t}} a b l_{l_{i t}^{\prime} b_{i t}}\left(b_{i t}\right) .
$$

Требуется доказать, что выполняется система равенств

$$
\left\{\begin{array}{l}
\text { НОД }\left(d_{1}, p^{m_{1}-m_{2}} d_{2}, \ldots, p^{m_{1}-m_{t}} d_{t}, p^{m_{1}}, n-1\right)= \\
=\text { НОД }\left(d_{1}^{\prime}, p^{m_{1}-m_{2}} d_{2}^{\prime}, \ldots, p^{m_{1}-m_{t}} d_{t}^{\prime}, p^{m_{1}}, n-1\right), \\
\text { НОД }\left(d_{1}, d_{2}, p^{m_{2}-m_{3}} d_{3}, \ldots, p^{m_{2}-m_{t}} d_{t}, p^{m_{2}}, n-1\right)= \\
=\text { НОД }\left(d_{1}^{\prime}, d_{2}^{\prime}, p^{m_{2}-m_{3}} d_{3}^{\prime}, \ldots, p^{m_{2}-m_{t}} d_{t}^{\prime}, p^{m_{2}}, n-1\right), \\
\ldots \\
\text { НОД }\left(d_{1}, \ldots, d_{t-1}, p^{m_{t-1}-m_{t}} d_{t}, p^{m_{t-1}}, n-1\right)= \\
=\text { НОД }\left(d_{1}^{\prime}, \ldots, d_{t-1}^{\prime}, p^{m_{t-1}-m_{t}} d_{t}^{\prime}, p^{m_{t-1}}, n-1\right), \\
\text { НОД }\left(d_{1}, \ldots, d_{t-1}, d_{t}, p^{m_{t}}, n-1\right)=\operatorname{HОД~}\left(d_{1}^{\prime}, \ldots, d_{t-1}^{\prime}, d_{t}^{\prime}, p^{m_{t}}, n-1\right),
\end{array}\right.
$$

где $d_{j}^{\prime}=$ НОД $\left(l_{1 j}^{\prime}, \ldots, l_{r_{j} j}^{\prime}\right)$, для всех $j=1, \ldots, t$.

Согласно теореме 1 найдутся изоморфизм $\sigma$ из $A$ в $B$ и элемент $u \in B$ такие, что верно (2), где $d=\sum_{j=1}^{t} \sum_{i=1}^{r_{j}} l_{i j} a_{i j}, d^{\prime}=\sum_{j=1}^{t} \sum_{i=1}^{r_{j}} l_{i j}^{\prime} b_{i j}$. Пусть $u=$ $\sum_{j=1}^{t} \sum_{i=1}^{r_{j}} u_{i j} b_{i j}$. Элементы $c_{i j}=\sigma\left(a_{i j}\right)$, где $i=1, \ldots, r_{j}$ при $j=1, \ldots, t$, будут базисом в группе $B$, а значит, имеется автоморфизм $\tau$ группы $B$, переводящий базисные элементы $b_{11}, \ldots, b_{r_{t} t}$ в базис $c_{11}, \ldots, c_{r_{t} t}$, т.е. $\tau\left(b_{i j}\right)=c_{i j}$. Тогда из $(2)$ имеем

$$
\sigma(d)=\sum_{j=1}^{t} \sum_{i=1}^{r_{j}} l_{i j} c_{i j}=\sum_{j=1}^{t} \sum_{i=1}^{r_{j}}\left((n-1) u_{i j}+l_{i j}^{\prime}\right) b_{i j}
$$

кроме того,

$$
\sigma(d)=\sum_{j=1}^{t} \sum_{i=1}^{r_{j}} l_{i j} c_{i j}=\sum_{j=1}^{t} \sum_{i=1}^{r_{j}} l_{i j} \tau\left(b_{i j}\right)=\tau\left(\sum_{j=1}^{t} \sum_{i=1}^{r_{j}} l_{i j} b_{i j}\right),
$$

тогда $\tau\left(\sum_{j=1}^{t} \sum_{i=1}^{r_{j}} l_{i j} b_{i j}\right)=\sum_{j=1}^{t} \sum_{i=1}^{r_{j}}\left((n-1) u_{i j}+l_{i j}^{\prime}\right) b_{i j}$, а значит, элемент $\sum_{j=1}^{t} \sum_{i=1}^{r_{j}} l_{i j} b_{i j}$ лежит в орбите элемента $\sum_{j=1}^{t} \sum_{i=1}^{r_{j}}\left((n-1) u_{i j}+l_{i j}^{\prime}\right) b_{i j}$ при действии группы автоморфизмов Aut $B$ на $B$.

Лемма 1. Пусть $G=\sum_{s=1}^{k} G_{s}-$ прямая сумма абелевых р-групп $G_{s}$, где каждая группа $G_{s}=\sum_{i=1}^{n_{s}}\left(a_{i s}\right)$ - прямая сумма ииклических групп $\left(a_{i s}\right)$ одного и того же порядка $p^{\alpha_{s}}$ и $\alpha_{1}>\alpha_{2}>\ldots>\alpha_{k}, p$ - простое число. Каждая орбита элемента 
$\sum_{s=1}^{k} \sum_{i=1}^{n_{s}} l_{i s}^{\prime} a_{i s}$ при действии группы автоморфизмов Aиt $G$ на $G$ состоит из всех элементов $\sum_{s=1}^{k} \sum_{i=1}^{n_{s}} l_{i s} a_{i s}$, для которьх

$$
\left\{\begin{array}{l}
\text { НОД }\left(d_{1}^{\prime}, p^{\alpha_{1}-\alpha_{2}} d_{2}^{\prime}, \ldots, p^{\alpha_{1}-\alpha_{k}} d_{k}^{\prime}, p^{\alpha_{1}}\right)=\operatorname{HОД}\left(d_{1}, p^{\alpha_{1}-\alpha_{2}} d_{2}, \ldots, p^{\alpha_{1}-\alpha_{k}} d_{k}, p^{\alpha_{1}}\right), \\
\text { НОД }\left(d_{1}^{\prime}, d_{2}^{\prime}, p^{\alpha_{2}-\alpha_{3}} d_{3}^{\prime}, \ldots, p^{\alpha_{2}-\alpha_{k}} d_{k}^{\prime}, p^{\alpha_{2}}\right)=\operatorname{HОД}\left(d_{1}, d_{2}, p^{\alpha_{2}-\alpha_{3}} d_{3}, \ldots, p^{\alpha_{2}-\alpha_{k}} d_{k}, p^{\alpha_{2}}\right), \\
\ldots \\
\text { НОД}\left(d_{1}^{\prime}, d_{2}^{\prime}, \ldots, d_{k-1}^{\prime}, p^{\alpha_{k-1}-\alpha_{k}} d_{k}^{\prime}, p^{\alpha_{k-1}}\right)=\operatorname{HОД}\left(d_{1}, d_{2}, \ldots, d_{k-1}, p^{\alpha_{k-1}-\alpha_{k}} d_{k}, p^{\alpha_{k-1}}\right), \\
\text { НОД}\left(d_{1}^{\prime}, d_{2}^{\prime}, \ldots, d_{k-1}^{\prime}, d_{k}^{\prime}, p^{\alpha_{k}}\right)=\operatorname{HОД}\left(d_{1}, d_{2}, \ldots, d_{k-1}, d_{k}, p^{\alpha_{k}}\right),
\end{array}\right.
$$

где $d_{s}^{\prime}=$ НОД $\left(l_{1 s}^{\prime}, \ldots, l_{n_{s} s}^{\prime}\right), d_{s}=$ НОД $\left(l_{1 s}, \ldots, l_{n_{s} s}\right)$ для всех $s=1, \ldots, k$.

Доказательство леммы 1. Покажем вначале, что каждый элемент $\sum_{s=1}^{k} \sum_{i=1}^{n_{s}} l_{i s} a_{i s}$, удовлетворяющий условию (9), принадлежит орбите элемента $\sum_{s=1}^{k} \sum_{i=1}^{n_{s}} l_{i s}^{\prime} a_{i s}$ при действии группы автоморфизмов Aut $G$ на $G$. Для этого будем искать автоморфизм $\sigma$ группы $G$, для которого $\sigma\left(\sum_{s=1}^{k} \sum_{i=1}^{n_{s}} l_{i s}^{\prime} a_{i s}\right)=$ $\sum_{s=1}^{k} \sum_{i=1}^{n_{s}} l_{i s} a_{i s}$.

Пусть в системе (9) правая и левая части $s$-го равенства равны $p^{\delta_{s}}(s=1, \ldots, k)$. Полагаем $l_{i j}^{\prime}=p^{\beta_{i j}} m_{i j}^{\prime}, l_{i j}=p^{\gamma_{i j}} m_{i j}$, где $m_{i j}^{\prime}$ и $m_{i j}$ не делятся на $p$. Кроме того, пусть $d_{s}^{\prime}=p^{\beta_{s}} q_{s}^{\prime}, d_{s}=p^{\gamma_{s}} q_{s}$, где $q_{s}^{\prime}$ и $q_{s}$ не делятся на $p$. Каждое число первого равенства из системы (9) делим на $p^{\delta_{1}}$, получим два набора чисел

$$
\begin{aligned}
& p^{\beta_{1}-\delta_{1}} q_{1}^{\prime}, p^{\alpha_{1}-\alpha_{2}+\beta_{2}-\delta_{1}} q_{2}^{\prime}, \ldots, p^{\alpha_{1}-\alpha_{k}+\beta_{k}-\delta_{1}} q_{k}^{\prime} \\
& p^{\gamma_{1}-\delta_{1}} q_{1}, p^{\alpha_{1}-\alpha_{2}+\gamma_{2}-\delta_{1}} q_{2}, \ldots, p^{\alpha_{1}-\alpha_{k}+\gamma_{k}-\delta_{1}} q_{k}
\end{aligned}
$$

В последовательности чисел (10) есть числа, взаимно простые с $p$. Среди таких чисел выбираем крайнее справа, пусть это будет $q_{t_{1}}^{\prime}=m_{r_{1} t_{1}}^{\prime}$, где $1 \leqslant t_{1} \leqslant k, 1 \leqslant$ $r_{1} \leqslant n_{t_{1}}$. Аналогично среди чисел последовательности (11) есть числа, взаимно простые с $p$. Среди таких чисел выбираем крайнее справа, пусть это будет $q_{v_{1}}$, где $1 \leqslant v_{1} \leqslant k$. Кроме того, пусть $q_{v_{1}}=m_{u_{1} v_{1}}$, где $1 \leqslant u_{1} \leqslant n_{v_{1}}$. Покажем, что $t_{1}=v_{1}$. От противного, пусть, например, $t_{1}>v_{1}$. Тогда $\delta_{1}=\alpha_{1}-\alpha_{t_{1}}+\beta_{t_{1}}$ и $\delta_{1}$ является наименьшим среди чисел

$$
\beta_{1}, \alpha_{1}-\alpha_{2}+\beta_{2}, \ldots, \alpha_{1}-\alpha_{k}+\beta_{k}
$$

Заметим, что $\delta_{2}-$ наименьшее число среди чисел

$$
\beta_{1}, \beta_{2}, \alpha_{2}-\alpha_{3}+\beta_{3}, \ldots, \alpha_{2}-\alpha_{k}+\beta_{k},
$$

и так как $t_{1}>1$ (это следует из предположения $t_{1}>v_{1}$ ), то $\delta_{2}$ выбирается как наименьшее среди чисел $\beta_{2}, \alpha_{2}-\alpha_{3}+\beta_{3}, \ldots, \alpha_{2}-\alpha_{k}+\beta_{k}$, которые получаются соответственно из набора чисел $\alpha_{1}-\alpha_{2}+\beta_{2}, \alpha_{1}-\alpha_{3}+\beta_{3}, \ldots, \alpha_{1}-\alpha_{k}+\beta_{k}$ путем вычитания из каждого числа одного и того же числа $\alpha_{1}-\alpha_{2}$, а значит, $\delta_{2}=\alpha_{2}-$ $\alpha_{t_{1}}+\beta_{t_{1}}$. Аналогично, $\delta_{3}=\alpha_{3}-\alpha_{t_{1}}+\beta_{t_{1}}$, и так далее, $\delta_{t_{1}-1}=\alpha_{t_{1}-1}-\alpha_{t_{1}}+\beta_{t_{1}}$, $\delta_{t_{1}}=\beta_{t_{1}}$. Отсюда получим равенства

$$
\delta_{j}=\alpha_{j}-\alpha_{j+1}+\delta_{j+1}, j=1, \ldots, t_{1}-1 .
$$

Кроме того, $\delta_{1}$ является наименьшим среди чисел $\gamma_{1}, \alpha_{1}-\alpha_{2}+\gamma_{2}, \ldots, \alpha_{1}-\alpha_{k}+\gamma_{k}$. Если $v_{1}=1$, то $\delta_{1}=\gamma_{1}$ и по выбору $q_{v_{1}}$ (крайнее справа) имеем

$$
\gamma_{1}<\alpha_{1}-\alpha_{j}+\gamma_{j}, j=2, \ldots, k \text {. }
$$


Заметим также, что $\delta_{2}-$ наименьшее число среди чисел $\gamma_{1}, \gamma_{2}, \alpha_{2}-\alpha_{3}+\gamma_{3}, \ldots, \alpha_{2}-$ $\alpha_{k}+\gamma_{k}$ и $\delta_{2}<\gamma_{1}$, так как если бы $\delta_{2}=\gamma_{1}$, то имели бы $\delta_{2}=\delta_{1}$, чего не может быть (см. выше). Тогда $\delta_{2}$ выбирается как минимум среди чисел $\gamma_{2}, \alpha_{2}-\alpha_{3}+\gamma_{3}, \ldots, \alpha_{2}-$ $\alpha_{k}+\gamma_{k}$. Пусть $\delta_{2}=\alpha_{2}-\alpha_{w_{1}}+\gamma_{w_{1}}$, где $2 \leqslant w_{1} \leqslant k$, тогда из (12) имеем $\gamma_{1}=$ $\delta_{1}=\alpha_{1}-\alpha_{2}+\delta_{2}=\alpha_{1}-\alpha_{w_{1}}+\gamma_{w_{1}}-$ противоречие с (13). Если же $v_{1}>1$, то $\delta_{1}=\alpha_{1}-\alpha_{v_{1}}+\gamma_{v_{1}}$, и вновь по выбору $q_{v_{1}}$ (крайнее справа) имеем

$$
\alpha_{1}-\alpha_{v_{1}}+\gamma_{v_{1}}<\alpha_{1}-\alpha_{j}+\gamma_{j}, j=v_{1}+1, \ldots, k .
$$

Далее, как и выше, получим $\delta_{2}=\alpha_{2}-\alpha_{v_{1}}+\gamma_{v_{1}}, \delta_{3}=\alpha_{3}-\alpha_{v_{1}}+\gamma_{v_{1}}, \ldots, \delta_{v_{1}-1}=$ $\alpha_{v_{1}-1}-\alpha_{v_{1}}+\gamma_{v_{1}}, \delta_{v_{1}}=\gamma_{v_{1}}$. Заметим также, что $\delta_{v_{1}+1}-$ наименьшее число среди чисел

$$
\gamma_{1}, \ldots, \gamma_{v_{1}}, \gamma_{v_{1}+1}, \alpha_{v_{1}+1}-\alpha_{v_{1}+2}+\gamma_{v_{1}+2}, \ldots, \alpha_{v_{1}+1}-\alpha_{k}+\gamma_{k}
$$

и $\delta_{v_{1}+1}<\gamma_{v_{1}}$, так как если бы выполнялось равенство $\delta_{v_{1}+1}=\gamma_{v_{1}}$, то имели бы $\delta_{v_{1}+1}=\delta_{v_{1}}$, чего не может быть (см. выше). Тогда $\delta_{v_{1}+1}$ выбирается как минимум среди чисел

$$
\gamma_{v_{1}+1}, \alpha_{v_{1}+1}-\alpha_{v_{1}+2}+\gamma_{v_{1}+2}, \ldots, \alpha_{v_{1}+1}-\alpha_{k}+\gamma_{k} .
$$

Полагаем $\delta_{v_{1}+1}=\alpha_{v_{1}+1}-\alpha_{w_{1}}+\gamma_{w_{1}}$, где $v_{1}+1 \leqslant w_{1} \leqslant k$, тогда из (12) следует, что $\gamma_{v_{1}}=\delta_{v_{1}}=\alpha_{v_{1}}-\alpha_{v_{1}+1}+\delta_{v_{1}+1}=\alpha_{v_{1}}-\alpha_{w_{1}}+\gamma_{w_{1}}-$ противоречие с (14). Итак, мы доказали равенство $t_{1}=v_{1}$.

Далее, каждое число равенства под номером $t_{1}+1$ из системы $(9)$ делим на $p^{\delta_{t_{1}+1}}$, получим два набора чисел

$p^{\beta_{1}-\delta_{t_{1}+1}} q_{1}^{\prime}, \ldots, p^{\beta_{t_{1}+1}-\delta_{t_{1}+1}} q_{t_{1}+1}^{\prime}, p^{\alpha_{t_{1}+1}-\alpha_{t_{1}+2}+\beta_{t_{1}+2}-\delta_{t_{1}+1}} q_{t_{1}+2}^{\prime}, \ldots, p^{\alpha_{t_{1}+1}-\alpha_{k}+\beta_{k}-\delta_{t_{1}+1}} q_{k}^{\prime}$,

$p^{\gamma_{1}-\delta_{t_{1}+1}} q_{1}, \ldots, p^{\gamma_{t_{1}+1}-\delta_{t_{1}+1}} q_{t_{1}+1}, p^{\alpha_{t_{1}+1}-\alpha_{t_{1}+2}+\gamma_{t_{1}+2}-\delta_{t_{1}+1}} q_{t_{1}+2}, \ldots, p^{\alpha_{t_{1}+1}-\alpha_{k}+\gamma_{k}-\delta_{t_{1}+1}} q_{k}$.

В последовательности чисел (15) есть числа, взаимно простые с $p$. Среди таких чисел выбираем крайнее справа, пусть это будет $q_{t_{2}}^{\prime}$, где $t_{1}+1 \leqslant t_{2} \leqslant k$. Кроме того, пусть $q_{t_{2}}^{\prime}=m_{r_{2} t_{2}}^{\prime}$, Аналогично среди чисел последовательности (16) есть числа, взаимно простые с $p$. Среди таких чисел выбираем крайнее справа, пусть это будет $q_{v_{2}}$, где $t_{1}+1 \leqslant v_{2} \leqslant k$. Кроме того, пусть $q_{v_{2}}=m_{u_{2} v_{2}}$, где $1 \leqslant u_{2} \leqslant n_{v_{2}}$. Аналогично доказывается равенство $t_{2}=v_{2}$.

Продолжая так двигаться дальше по системе равенств (9) сверху вниз, на некотором $m$-м шаге мы каждое число равенства под номером $t_{m-1}+1$ из системы (9) делим на $p^{\delta_{t_{m-1}+1}}$, получая два набора чисел

$$
\begin{gathered}
p^{\beta_{1}-\delta_{t_{m-1}+1}} q_{1}^{\prime}, \ldots, p^{\beta_{t_{m-1}+1}-\delta_{t_{m-1}+1}} q_{t_{m-1}+1}^{\prime}, \\
p^{\alpha_{t_{m-1}+1}-\alpha_{t_{m-1}+2}+\beta_{t_{m-1}+2}-\delta_{t_{m-1}+1}} q_{t_{m-1}+2}^{\prime}, \ldots, p^{\alpha_{t_{m-1}+1}-\alpha_{k}+\beta_{k}-\delta_{t_{m-1}+1}} q_{k}^{\prime}, \\
p^{\gamma_{1}-\delta_{t_{m-1}+1}} q_{1}, \ldots, p^{\gamma_{t_{m-1}+1}-\delta_{t_{m-1}+1}} q_{t_{m-1}+1}, \\
p^{\alpha_{t_{m-1}+1}-\alpha_{t_{m-1}+2}+\gamma_{t_{m-1}+2}-\delta_{t_{m-1}+1}} q_{t_{m-1}+2}, \ldots, p^{\alpha_{t_{m-1}+1}-\alpha_{k}+\gamma_{k}-\delta_{t_{m-1}+1}} q_{k} .
\end{gathered}
$$

В последовательности чисел (17) есть числа, взаимно простые с $p$. Среди таких чисел выбираем крайнее справа, пусть это будет $q_{t_{m}}^{\prime}$, где $t_{m}=k$. Кроме того, пусть $q_{k}^{\prime}=m_{r_{m} k}^{\prime}$, где $1 \leqslant r_{m} \leqslant n_{k}$. Аналогично, среди чисел последовательности (18) есть числа, взаимно простые с $p$. Среди таких чисел выбираем крайнее справа, пусть это будет $q_{v_{m}}=m_{u_{m} v_{m}}$, где $t_{m-1}+1 \leqslant v_{m} \leqslant k, 1 \leqslant u_{m} \leqslant n_{v_{m}}$. Аналогично доказывается равенство $v_{m}=k$. 
Для каждого $l=1, \ldots, m$ обозначим $b_{i j}=a_{i j}$, где $j$ пробегает значения $t_{l-1}+$ $1, \ldots, t_{l}$ (здесь и дальше $t_{0}=0$ ), а вместе с заданным $j$ индекс $i$, в свою очередь, пробегает значения $1, \ldots, n_{j}$, кроме $i=u_{l}$ и $i=r_{l}$ при $j=t_{l}$, и $b_{u_{l} t_{l}}=a_{r_{l} t_{l}}, b_{r_{l} t_{l}}=$ $\sum_{j=t_{l-1}+1}^{t_{l}} \sum_{i=1}^{n_{j}} p^{\alpha_{j}-\alpha_{t}} q_{i j} a_{i j}$, где $q_{i j}$ - решение сравнения

$$
m_{r_{l} t_{l}}^{\prime} x \equiv p^{\gamma_{i j}-\delta_{j}} m_{i j}-p^{\beta_{i j}-\delta_{j}} m_{i j}^{\prime} \quad\left(\bmod p^{\alpha_{j}-\delta_{j}}\right),
$$

где $j$ пробегает значения $1, \ldots, t_{l}$, а вместе с заданным $j$ индекс $i$ в свою очередь пробегает значения $1, \ldots, n_{j}$, кроме $i=u_{l}$ и $i=r_{l}$ при $j=t_{l}$, и $q_{r_{l} t_{l}}$ - решение сравнения

$$
m_{r_{l} t_{l}}^{\prime} x \equiv m_{r_{l} t_{l}}-m_{u_{l} t_{l}}^{\prime} \quad\left(\bmod p^{\alpha_{t_{l}}-\delta_{t_{l}}}\right),
$$

$q_{u_{l} t_{l}}$ - решение сравнения

$$
m_{r_{l} t_{l}}^{\prime} x \equiv m_{u_{l} t_{l}} \quad\left(\bmod p^{\alpha_{t_{l}}-\delta_{t_{l}}}\right)
$$

Из (19) следует

$$
l_{i j}^{\prime}+l_{r_{l} t_{l}}^{\prime} p^{\alpha_{j}-\alpha_{t}} q_{i j} \equiv l_{i j} \quad\left(\bmod p^{\alpha_{j}}\right) .
$$

Из (20) следует

$$
l_{u_{l} t_{l}}^{\prime}+l_{r_{l} t_{l}}^{\prime} q_{r_{l} t_{l}} \equiv l_{r_{l} t_{l}} \quad\left(\bmod p^{\alpha_{t_{l}}}\right),
$$

а из (21) следует

$$
l_{r_{l} t_{l}}^{\prime} q_{u_{l} t_{l}} \equiv l_{u_{l} t_{l}} \quad\left(\bmod p^{\alpha_{t_{l}}}\right) .
$$

Покажем теперь, что все так найденные элементы $b_{i j}$ образуют базис группы $G$. Действительно, если верно равенство $\sum_{l=1}^{m} \sum_{j=t_{l-1}+1}^{t_{l}} \sum_{i=1}^{n_{j}} h_{i j} b_{i j}=0$, то верно равенство

$$
\begin{aligned}
\sum_{l=1}^{m}\left(\sum_{j=t_{l-1}+1}^{t_{l}-1} \sum_{i=1}^{n_{j}} h_{i j} a_{i j}+\right. & \sum_{i=1, i \neq r_{l}, i \neq u_{l}}^{n_{t_{l}}} h_{i t_{l}} a_{i t_{l}}+ \\
& \left.+h_{r_{l} t_{l}}\left(\sum_{j=t_{l-1}+1}^{t_{l}} \sum_{i=1}^{n_{j}} p^{\alpha_{j}-\alpha_{t_{l}}} q_{i j} a_{i j}\right)+h_{u_{l} t_{l}} a_{r_{l} t_{l}}\right)=0,
\end{aligned}
$$

или

$$
\begin{aligned}
\sum_{l=1}^{m}\left(\left(h_{r_{l} t_{l}} q_{r_{l} t_{l}}+h_{u_{l} t_{l}}\right) a_{r_{l} t_{l}}+h_{r_{l} t_{l}} q_{u_{l} t_{l}} a_{u_{l} t_{l}}\right. & +\sum_{j=t_{l-1}+1}^{t_{l}-1} \sum_{i=1}^{n_{j}}\left(h_{i j}+h_{r_{l} t_{l}} p^{\alpha_{j}-\alpha_{t_{l}}} q_{i j}\right) a_{i j}+ \\
& \left.+\sum_{i=1, i \neq r_{l}, i \neq u_{l}}^{n_{t_{l}}}\left(h_{i t_{l}}+h_{r_{l} t_{l}} q_{i t_{l}}\right) a_{i t_{l}}\right)=0 .
\end{aligned}
$$

Но все элементы $a_{i j}$ образуют базис группы $G$, значит, для всех $l=1, \ldots, m$ будем иметь $h_{i j}+h_{r_{l} t_{l}} p^{\alpha_{j}-\alpha_{t}} q_{i j} \equiv 0\left(\bmod p^{\alpha_{j}}\right)$, где $j$ пробегает значения $t_{l-1}+1, \ldots, t_{l}$, а вместе с заданным $j$ индекс $i$, в свою очередь, пробегает значения $1, \ldots, n_{j}$, кроме $i=r_{l}$ и $i=u_{l}$ при $j=t_{l}$, и $h_{r_{l} t_{l}} q_{r_{l} t_{l}}+h_{u_{l} t_{l}} \equiv 0\left(\bmod p^{\alpha_{t_{l}}}\right), h_{r_{l} t_{l}} q_{u_{l} t_{l}} \equiv 0\left(\bmod p^{\alpha_{t_{l}}}\right)$. Так как $q_{u_{l} t_{l}}$ взаимно просто с $p$ (это следует из (21) и взаимной простоты чисел $m_{r_{l} t_{l}}^{\prime}$ и $m_{u_{l} t_{l}}$ с $\left.p\right)$, то из последнего сравнения имеем $h_{r_{l} t_{l}} \equiv 0\left(\bmod p^{\alpha_{t_{l}}}\right)$. Тогда $h_{u_{l} t_{l}} \equiv 0\left(\bmod p^{\alpha_{t_{l}}}\right)$ и $h_{r_{l} t_{l}} p^{\alpha_{j}-\alpha_{t_{l}}} \equiv 0\left(\bmod p^{\alpha_{j}}\right)$, откуда $h_{i j} \equiv 0\left(\bmod p^{\alpha_{j}}\right)$. Таким образом, мы доказали, что все элементы $b_{i j}$ образуют базис группы $G$. 
Выбираем автоморфизм $\sigma$ группы $G$, удовлетворяющий условию: для всех $l=$ $1, \ldots, m$ выполняется равенство $\sigma\left(a_{i j}\right)=b_{i j}$, где $j$ пробегает значения $t_{l-1}+1, \ldots, t_{l}$, а при заданном $j$ индекс $i$, в свою очередь, пробегает значения $1, \ldots, n_{j}$. Тогда, используя $(22),(23)$ и $(24)$, получим

$$
\begin{gathered}
\sigma\left(\sum_{j=1}^{k} \sum_{i=1}^{n_{j}} l_{i j}^{\prime} a_{i j}\right)=\sum_{j=1}^{k} \sum_{i=1}^{n_{j}} l_{i j}^{\prime} \sigma\left(a_{i j}\right)=\sum_{j=1}^{k} \sum_{i=1}^{n_{j}} l_{i j}^{\prime} b_{i j}= \\
=\sum_{l=1}^{m}\left(\sum_{j=t_{l-1}+1}^{t_{l}-1} \sum_{i=1}^{n_{j}} l_{i j}^{\prime} a_{i j}+\sum_{i=1, i \neq r_{l}, i \neq u_{l}}^{n_{t_{l}}} l_{i t_{l}}^{\prime} a_{i t_{l}}+l_{u_{l} t_{l}}^{\prime} a_{r_{l} t_{l}}+\right. \\
\left.+l_{r_{l} t_{l}}^{\prime}\left(\sum_{j=t_{l-1}+1}^{t_{l}} \sum_{i=1}^{n_{j}} p^{\alpha_{j}-\alpha_{t_{l}}} q_{i j} a_{i j}\right)\right)= \\
=\sum_{l=1}^{m}\left(\sum_{j=t_{l-1}+1}^{t_{l}-1} \sum_{i=1}^{n_{j}}\left(l_{i j}^{\prime}+l_{r_{l} t_{l}}^{\prime} p^{\alpha_{j}-\alpha_{t_{l}}} q_{i j}\right) a_{i j}+\right. \\
\left.+\sum_{i=1, i \neq r_{l}, i \neq u_{l}}^{n_{t_{l}}}\left(l_{i t_{l}}^{\prime}+l_{r_{l} t_{l}}^{\prime} q_{i t_{l}}\right) a_{i t_{l}}+\left(l_{u_{l} t_{l}}^{\prime}+l_{r_{l} t_{l}}^{\prime} q_{r_{l} t_{l}}\right) a_{r_{l} t_{l}}+l_{r_{l} t_{l}}^{\prime} q_{u_{l} t_{l}} a_{u_{l} t_{l}}\right)= \\
\sum_{l=1}^{m}\left(\sum_{j=t_{l-1}+1}^{t_{l}-1} \sum_{i=1}^{n_{j}} l_{i j} a_{i j}+\sum_{i=1, i \neq r_{l}, i \neq u_{l}}^{n_{t_{l}}} l_{i t_{l}} a_{i t_{l}}+l_{r_{l} t_{l}} a_{r_{l} t_{l}}+l_{u_{l} t_{l}} a_{u_{l} t_{l}}\right)=\sum_{s=1}^{k} \sum_{i=1}^{n_{s}} l_{i s} a_{i s} .
\end{gathered}
$$

Обратно. Покажем теперь, что если элемент $\sum_{s=1}^{k} \sum_{i=1}^{n_{s}} l_{i s} a_{i s}$ принадлежит орбите элемента $\sum_{s=1}^{k} \sum_{i=1}^{n_{s}} l_{i s}^{\prime} a_{i s}$ при действии группы автоморфизмов Aut $G$ на $G$, то для этого элемента выполнены условия (9).

Известно (см., например, $\S 21,[8])$, что кольцо эндоморфизмов End $G$ изоморфно кольцу $M$ целочисленных матриц $\left(y_{i s}^{j t}\right)$ порядка $n_{1}+\ldots+n_{k}$, где $1 \leqslant s, t \leqslant k$, а при заданных $s$ и $t$ для индексов $i, j$ выполнены неравенства $\sum_{r=1}^{s-1} n_{r}+1 \leqslant i \leqslant \sum_{r=1}^{s} n_{r}$ и $\sum_{r=1}^{t-1} n_{r}+1 \leqslant j \leqslant \sum_{r=1}^{t} n_{r}$ (здесь и дальше $n_{0}=0$ при $s=1$ и $t=1$ ), нижняя пара индексов $i s$ определяет номер строки $\sum_{r=1}^{s-1} n_{r}+i$, а верхняя пара индексов $j t$ определяет номер столбца $\sum_{r=1}^{t-1} n_{r}+j$, на пересечении которых стоит элемент $y_{i s}^{j t}$, и

$$
y_{i s}^{j t}= \begin{cases}x_{i s}^{j t}, & \text { если } s<t \text { или } s=t \text { вместе с } i<j, \text { где } 0 \leqslant x_{i s}^{j t}<p^{\alpha_{t}}, \\ p^{\alpha_{t}-\alpha_{s}} x_{i s}^{j t}, & \text { если } s>t \text { или } s=t \text { вместе с } i \geqslant j, \text { где } 0 \leqslant x_{i s}^{j t}<p^{\alpha_{s}},\end{cases}
$$

с операциями сложения матриц $\left(y_{i s}^{j t}\right)+\left(y_{i s}^{\prime j t}\right)=\left(y_{i s}^{j t}+y_{i s}^{\prime j t}\left(\bmod p^{\alpha_{t}}\right)\right)$, и умножения матриц $\left(y_{i s}^{j t}\right) \times\left(y_{i s}^{\prime j t}\right)=\left(\sum_{r=1}^{k} \sum_{v=1}^{n_{r}} y_{i s}^{v r} \cdot y_{v r}^{\prime j t}\left(\bmod p^{\alpha_{t}}\right)\right)$. Каждому автоморфизму $\sigma$ группы $G$ соответствует (при указанном изоморфизме) обратимая матрица $\left(y_{i s}^{j t}\right)$ из кольца $M$, и $\sigma$ действует по правилу: если $g \in G$ и $g=\sum_{s=1}^{k} \sum_{i=1}^{n_{s}} q_{i s} a_{i s}$, то

$$
\sigma(g)=\sum_{t=1}^{k} \sum_{j=1}^{n_{t}}\left(\sum_{s=1}^{k} \sum_{i=1}^{n_{s}} q_{i s} y_{i s}^{j t}\right) a_{j t}
$$

Пусть $\sigma\left(\sum_{s=1}^{k} \sum_{i=1}^{n_{s}} l_{i s}^{\prime} a_{i s}\right)=\sum_{s=1}^{k} \sum_{i=1}^{n_{s}} l_{i s} a_{i s}$ для некоторого автоморфизма $\sigma$ группы $G$, который задается целочисленной матрицей $\left(y_{i s}^{j t}\right)$. Тогда, используя (25) 
и (26), получим систему сравнений

$$
\left\{\begin{array}{l}
\sum_{s=1}^{k} \sum_{i=1}^{n_{s}} l_{i s}^{\prime} p^{\alpha_{1}-\alpha_{s}} x_{i s}^{11} \equiv l_{11} \quad\left(\bmod p^{\alpha_{1}}\right) \\
\cdots \\
\sum_{s=1}^{k} \sum_{i=1}^{n_{s}} l_{i s}^{\prime} p^{\alpha_{1}-\alpha_{s}} x_{i s}^{n_{1} 1} \equiv l_{n_{1} 1} \quad\left(\bmod p^{\alpha_{1}}\right) \\
\sum_{i=1}^{n_{1}} l_{i 1}^{\prime} x_{i 1}^{12}+\sum_{s=2}^{k} \sum_{i=1}^{n_{s}} l_{i s}^{\prime} p^{\alpha_{2}-\alpha_{s}} x_{i s}^{12} \equiv l_{12} \quad\left(\bmod p^{\alpha_{2}}\right) \\
\cdots \\
\sum_{i=1}^{n_{1}} l_{i 1}^{\prime} x_{i 1}^{n_{2} 2}+\sum_{s=2}^{k} \sum_{i=1}^{n_{s}} l_{i s}^{\prime} p^{\alpha_{2}-\alpha_{s}} x_{i s}^{n_{2} 2} \equiv l_{n_{2} 2} \quad\left(\bmod p^{\alpha_{2}}\right) \\
\cdots \\
\sum_{s=1}^{k-1} \sum_{i=1}^{n_{s}} l_{i s}^{\prime} x_{i s}^{1 k-1}+\sum_{i=1}^{n_{k}} l_{i k}^{\prime} p^{\alpha_{k-1}-\alpha_{k}} x_{i k}^{1 k-1} \equiv l_{1 k-1} \quad\left(\bmod p^{\alpha_{k-1}}\right) \\
\cdots \\
\sum_{s=1}^{k-1} \sum_{i=1}^{n_{s}} l_{i s}^{\prime} x_{i s}^{n_{k-1} k-1}+\sum_{i=1}^{n_{k}} l_{i k}^{\prime} p^{\alpha_{k-1}-\alpha_{k}} x_{i k}^{n_{k-1} k-1} \equiv l_{n_{k-1} k-1} \quad\left(\bmod p^{\alpha_{k-1}}\right) \\
\sum_{s=1}^{k} \sum_{i=1}^{n_{s}} l_{i s}^{\prime} x_{i s}^{1 k} \equiv l_{1 k} \quad\left(\bmod p^{\alpha_{k}}\right) \\
\cdots \\
\sum_{s=1}^{k} \sum_{i=1}^{n_{s}} l_{i s}^{\prime} x_{i s}^{n_{k} k} \equiv l_{n_{k} k} \quad\left(\bmod p^{\alpha_{k}}\right)
\end{array}\right.
$$

Докажем первое равенство из (9). Из первых $n_{1}$ сравнений системы $(27)$ имеем

$$
\left\{\begin{array}{l}
l_{11}=\sum_{s=1}^{k} \sum_{i=1}^{n_{s}} l_{i s}^{\prime} p^{\alpha_{1}-\alpha_{s}} x_{i s}^{11}+p^{\alpha_{1}} q_{11}, \\
\ldots \\
l_{n_{1} 1}=\sum_{s=1}^{k} \sum_{i=1}^{n_{s}} l_{i s}^{\prime} p^{\alpha_{1}-\alpha_{s}} x_{i s}^{n_{1} 1}+p^{\alpha_{1}} q_{n_{1} 1}
\end{array}\right.
$$

для некоторых $q_{11}, \ldots, q_{n_{1} 1}$. Так как все числа $l_{i s}^{\prime} p^{\alpha_{1}-\alpha_{s}}$ из $(28)$ делятся на

$$
\text { НОД }\left(d_{1}^{\prime}, p^{\alpha_{1}-\alpha_{2}} d_{2}^{\prime}, \ldots, p^{\alpha_{1}-\alpha_{k}} d_{k}^{\prime}, p^{\alpha_{1}}\right) \text {, }
$$

TO

$$
\left\{\begin{array}{l}
\text { НОД }\left(d_{1}^{\prime}, p^{\alpha_{1}-\alpha_{2}} d_{2}^{\prime}, \ldots, p^{\alpha_{1}-\alpha_{k}} d_{k}^{\prime}, p^{\alpha_{1}}\right) \mid l_{11}, \\
\ldots \\
\text { НОД }\left(d_{1}^{\prime}, p^{\alpha_{1}-\alpha_{2}} d_{2}^{\prime}, \ldots, p^{\alpha_{1}-\alpha_{k}} d_{k}^{\prime}, p^{\alpha_{1}}\right) \mid l_{n_{1} 1},
\end{array}\right.
$$

а значит,

$$
\text { НОД }\left(d_{1}^{\prime}, p^{\alpha_{1}-\alpha_{2}} d_{2}^{\prime}, \ldots, p^{\alpha_{1}-\alpha_{k}} d_{k}^{\prime}, p^{\alpha_{1}}\right) \mid d_{1} .
$$

Из следующих $n_{2}$ сравнений системы (27) имеем

$$
\left\{\begin{array}{l}
l_{12}=\sum_{i=1}^{n_{1}} l_{i 1}^{\prime} x_{i 1}^{12}+\sum_{s=2}^{k} \sum_{i=1}^{n_{s}} l_{i s}^{\prime} p^{\alpha_{2}-\alpha_{s}} x_{i s}^{12}+p^{\alpha_{2}} q_{12} \\
\ldots \\
l_{n_{2} 2}=\sum_{i=1}^{n_{1}} l_{i 1}^{\prime} x_{i 1}^{n_{2} 2}+\sum_{s=2}^{k} \sum_{i=1}^{n_{s}} l_{i s}^{\prime} p^{\alpha_{2}-\alpha_{s}} x_{i s}^{n_{2} 2}+p^{\alpha_{2}} q_{n_{2} 2}
\end{array}\right.
$$

для некоторых $q_{12}, \ldots, q_{n_{2} 2}$. Так как все числа $l_{i 1}^{\prime}\left(i=1, \ldots, n_{1}\right)$ и $l_{i s}^{\prime} p^{\alpha_{2}-\alpha_{s}}$ (при $s=2, \ldots, k$ всякий раз $\left.i=1, \ldots, n_{s}\right)$ из $(31)$ делятся на

$$
\text { НОД }\left(d_{1}^{\prime}, d_{2}^{\prime}, p^{\alpha_{2}-\alpha_{3}} d_{3}^{\prime}, \ldots, p^{\alpha_{2}-\alpha_{k}} d_{k}^{\prime}, p^{\alpha_{2}}\right) \text {, }
$$

то

$$
\left\{\begin{array}{l}
\text { НОД }\left(d_{1}^{\prime}, d_{2}^{\prime}, p^{\alpha_{2}-\alpha_{3}} d_{3}^{\prime}, \ldots, p^{\alpha_{2}-\alpha_{k}} d_{k}^{\prime}, p^{\alpha_{2}}\right) \mid l_{12}, \\
\ldots \\
\text { НОД }\left(d_{1}^{\prime}, d_{2}^{\prime}, p^{\alpha_{2}-\alpha_{3}} d_{3}^{\prime}, \ldots, p^{\alpha_{2}-\alpha_{k}} d_{k}^{\prime}, p^{\alpha_{2}}\right) \mid l_{n_{2} 2},
\end{array}\right.
$$

а значит,

$$
\text { НОД }\left(d_{1}^{\prime}, d_{2}^{\prime}, p^{\alpha_{2}-\alpha_{3}} d_{3}^{\prime}, \ldots, p^{\alpha_{2}-\alpha_{k}} d_{k}^{\prime}, p^{\alpha_{2}}\right) \mid d_{2} \text {. }
$$


Из (33) имеем

$$
\text { НОД }\left(d_{1}^{\prime}, p^{\alpha_{1}-\alpha_{2}} d_{2}^{\prime}, \ldots, p^{\alpha_{1}-\alpha_{k}} d_{k}^{\prime}, p^{\alpha_{1}}\right) \mid p^{\alpha_{1}-\alpha_{2}} d_{2} \text {. }
$$

Из следующих $n_{3}$ сравнений системы (27) имеем

$$
\left\{\begin{array}{l}
l_{13}=\sum_{i=1}^{n_{1}} l_{i 1}^{\prime} x_{i 1}^{13}+\sum_{i=1}^{n_{2}} l_{i 2}^{\prime} x_{i 2}^{13}+\sum_{s=3}^{k} \sum_{i=1}^{n_{s}} l_{i s}^{\prime} p^{\alpha_{3}-\alpha_{s}} x_{i s}^{13}+p^{\alpha_{3}} q_{13} \\
\ldots \\
l_{n_{3} 3}=\sum_{i=1}^{n_{1}} l_{i 1}^{\prime} x_{i 1}^{n_{3} 3}+\sum_{i=1}^{n_{2}} l_{i 2}^{\prime} x_{i 2}^{n_{3} 3}+\sum_{s=3}^{k} \sum_{i=1}^{n_{s}} l_{i s}^{\prime} p^{\alpha_{3}-\alpha_{s}} x_{i s}^{n_{3} 3}+p^{\alpha_{3}} q_{n_{3} 3}
\end{array}\right.
$$

для некоторых $q_{13}, \ldots, q_{n_{3} 3}$. Так как все числа $l_{i 1}^{\prime}\left(i=1, \ldots, n_{1}\right), l_{i 2}^{\prime}\left(i=1, \ldots, n_{2}\right)$ и $l_{i s}^{\prime} p^{\alpha_{3}-\alpha_{s}}$ (при $s=3, \ldots, k$ всякий раз $\left.i=1, \ldots, n_{s}\right)$ из $(31)$ делятся на

$$
\text { НОД }\left(d_{1}^{\prime}, d_{2}^{\prime}, d_{3}^{\prime}, p^{\alpha_{3}-\alpha_{4}} d_{4}^{\prime}, \ldots, p^{\alpha_{3}-\alpha_{k}} d_{k}^{\prime}, p^{\alpha_{3}}\right),
$$

TO

$$
\left\{\begin{array}{l}
\text { НОД }\left(d_{1}^{\prime}, d_{2}^{\prime}, d_{3}^{\prime}, p^{\alpha_{3}-\alpha_{4}} d_{4}^{\prime}, \ldots, p^{\alpha_{3}-\alpha_{k}} d_{k}^{\prime}, p^{\alpha_{3}}\right) \mid l_{13}, \\
\ldots \\
\text { НОД }\left(d_{1}^{\prime}, d_{2}^{\prime}, d_{3}^{\prime}, p^{\alpha_{3}-\alpha_{4}} d_{4}^{\prime}, \ldots, p^{\alpha_{3}-\alpha_{k}} d_{k}^{\prime}, p^{\alpha_{3}}\right) \mid l_{n_{3} 3},
\end{array}\right.
$$

а значит,

$$
\text { НОД }\left(d_{1}^{\prime}, d_{2}^{\prime}, d_{3}^{\prime}, p^{\alpha_{3}-\alpha_{4}} d_{4}^{\prime}, \ldots, p^{\alpha_{3}-\alpha_{k}} d_{k}^{\prime}, p^{\alpha_{3}}\right) \mid d_{3} \text {. }
$$

Из (37) имеем

$$
\text { НОД }\left(d_{1}^{\prime}, d_{2}^{\prime}, p^{\alpha_{2}-\alpha_{3}} d_{3}^{\prime}, \ldots, p^{\alpha_{2}-\alpha_{k}} d_{k}^{\prime}, p^{\alpha_{2}}\right) \mid p^{\alpha_{2}-\alpha_{3}} d_{3},
$$

а из (38) следует, что

$$
\text { НОД }\left(d_{1}^{\prime}, p^{\alpha_{1}-\alpha_{2}} d_{2}^{\prime}, p^{\alpha_{1}-\alpha_{3}} d_{3}^{\prime}, \ldots, p^{\alpha_{1}-\alpha_{k}} d_{k}^{\prime}, p^{\alpha_{1}}\right) \mid p^{\alpha_{1}-\alpha_{3}} d_{3},
$$

Используя далее последовательно подсистемы сравнений по одному и тому же модулю $p^{\alpha_{s}}(s=1, \ldots, k)$, на которые разбита система $(27)$, получаем, что

$$
\text { НОД }\left(d_{1}^{\prime}, p^{\alpha_{1}-\alpha_{2}} d_{2}^{\prime}, \ldots, p^{\alpha_{1}-\alpha_{k}} d_{k}^{\prime}, p^{\alpha_{1}}\right) \mid p^{\alpha_{1}-\alpha_{s}} d_{s} \text { для всех } s=1, \ldots, k \text {. }
$$

Из (40) следует, что

$$
\text { НОД }\left(d_{1}^{\prime}, p^{\alpha_{1}-\alpha_{2}} d_{2}^{\prime}, \ldots, p^{\alpha_{1}-\alpha_{k}} d_{k}^{\prime}, p^{\alpha_{1}}\right) \mid \operatorname{НОД}\left(d_{1}, p^{\alpha_{1}-\alpha_{2}} d_{2}, \ldots, p^{\alpha_{1}-\alpha_{k}} d_{k}, p^{\alpha_{1}}\right) \text {. }
$$

В силу обратимости матрицы $\left(y_{i s}^{j t}\right)$ из кольца $M$ имеем аналогично

$$
\text { НОД }\left(d_{1}, p^{\alpha_{1}-\alpha_{2}} d_{2}, \ldots, p^{\alpha_{1}-\alpha_{k}} d_{k}, p^{\alpha_{1}}\right) \mid \operatorname{НОД}\left(d_{1}^{\prime}, p^{\alpha_{1}-\alpha_{2}} d_{2}^{\prime}, \ldots, p^{\alpha_{1}-\alpha_{k}} d_{k}^{\prime}, p^{\alpha_{1}}\right) \text {. }
$$

Из (41) и (42) следует первое равенство в (9).

Докажем второе равенство в (9). Из (30) имеем

$$
\text { НОД }\left(d_{1}^{\prime}, d_{2}^{\prime}, p^{\alpha_{2}-\alpha_{3}} d_{3}^{\prime}, \ldots, p^{\alpha_{2}-\alpha_{k}} d_{k}^{\prime}, p^{\alpha_{2}}\right) \mid d_{1} \text {. }
$$

Кроме того, из второй группы из $n_{2}$ сравнений системы (27) мы получили (33), а из третьей группы из $n_{3}$ сравнений системы (27) мы получили (38).

Используя далее последовательно подсистемы сравнений по одному и тому же модулю $p^{\alpha_{s}}(s=2, \ldots, k)$, на которые разбита система $(27)$, получаем, что

$$
\text { НОД }\left(d_{1}^{\prime}, d_{2}^{\prime}, p^{\alpha_{2}-\alpha_{3}} d_{3}^{\prime}, \ldots, p^{\alpha_{2}-\alpha_{k}} d_{k}^{\prime}, p^{\alpha_{2}}\right) \mid p^{\alpha_{2}-\alpha_{s}} d_{s} \text { для всех } s=2, \ldots, k .
$$


Из (43) и (44) следует, что

$$
\text { НОД }\left(d_{1}^{\prime}, d_{2}^{\prime}, p^{\alpha_{2}-\alpha_{3}} d_{3}^{\prime}, \ldots, p^{\alpha_{2}-\alpha_{k}} d_{k}^{\prime}, p^{\alpha_{2}}\right) \mid \operatorname{НОД}\left(d_{1}, d_{2}, p^{\alpha_{2}-\alpha_{3}} d_{3}, \ldots, p^{\alpha_{2}-\alpha_{k}} d_{k}, p^{\alpha_{2}}\right) \text {. }
$$

В силу обратимости матрицы $\left(y_{i s}^{j t}\right)$ из кольца $M$ имеем аналогично

$$
\text { НОД }\left(d_{1}, d_{2}, p^{\alpha_{2}-\alpha_{3}} d_{3}, \ldots, p^{\alpha_{2}-\alpha_{k}} d_{k}, p^{\alpha_{2}}\right) \mid \text { НОД }\left(d_{1}^{\prime}, d_{2}^{\prime}, p^{\alpha_{2}-\alpha_{3}} d_{3}^{\prime}, \ldots, p^{\alpha_{2}-\alpha_{k}} d_{k}^{\prime}, p^{\alpha_{2}}\right) \text {. }
$$

Из (45) и (46) следует второе равенство в (9).

Докажем третье равенство в (9). Из (30) имеем

$$
\text { НОД }\left(d_{1}^{\prime}, d_{2}^{\prime}, d_{3}^{\prime}, p^{\alpha_{3}-\alpha_{4}} d_{4}^{\prime}, \ldots, p^{\alpha_{3}-\alpha_{k}} d_{k}^{\prime}, p^{\alpha_{3}}\right) \mid d_{1} \text {, }
$$

а из (33) имеем

$$
\text { НОД }\left(d_{1}^{\prime}, d_{2}^{\prime}, d_{3}^{\prime}, p^{\alpha_{3}-\alpha_{4}} d_{4}^{\prime}, \ldots, p^{\alpha_{3}-\alpha_{k}} d_{k}^{\prime}, p^{\alpha_{3}}\right) \mid d_{2} \text {. }
$$

Кроме того, из третьей группы из $n_{3}$ сравнений системы (27) мы получили (38).

Используя далее последовательно подсистемы сравнений по одному и тому же модулю $p^{\alpha_{s}}(s=3, \ldots, k)$, на которые разбита система $(27)$, получаем

$$
\text { НОД }\left(d_{1}^{\prime}, d_{2}^{\prime}, d_{3}^{\prime}, p^{\alpha_{3}-\alpha_{4}} d_{4}^{\prime}, \ldots, p^{\alpha_{3}-\alpha_{k}} d_{k}^{\prime}, p^{\alpha_{3}}\right) \mid p^{\alpha_{3}-\alpha_{s}} d_{s} \text { для всех } s=3, \ldots, k \text {. }
$$

Из (47),(48) и (49) следует, что

НОД $\left(d_{1}^{\prime}, d_{2}^{\prime}, d_{3}^{\prime}, p^{\alpha_{3}-\alpha_{4}} d_{4}^{\prime}, \ldots, p^{\alpha_{3}-\alpha_{k}} d_{k}^{\prime}, p^{\alpha_{3}}\right) \mid$ НОД $\left(d_{1}, d_{2}, d_{3}, p^{\alpha_{3}-\alpha_{4}} d_{4}, \ldots, p^{\alpha_{3}-\alpha_{k}} d_{k}, p^{\alpha_{3}}\right)$.

В силу обратимости матрицы $\left(y_{i s}^{j t}\right)$ из кольца $M$ имеем аналогично

НОД $\left(d_{1}, d_{2}, d_{3}, p^{\alpha_{3}-\alpha_{4}} d_{4}, \ldots, p^{\alpha_{3}-\alpha_{k}} d_{k}, p^{\alpha_{3}}\right) \mid$ НОД $\left(d_{1}^{\prime}, d_{2}^{\prime}, d_{3}^{\prime}, p^{\alpha_{3}-\alpha_{4}} d_{4}^{\prime}, \ldots, p^{\alpha_{3}-\alpha_{k}} d_{k}^{\prime}, p^{\alpha_{3}}\right)$.

Из (50) и (51) имеем третье равенство в (9).

Продолжая так дальше, мы получим все равенства в (9). Лемма 1 доказана.

Продолжим доказательство теоремы 4. По лемме 1

$$
\left\{\begin{array}{l}
\text { НОД }\left(d_{1}^{\prime \prime}, p^{m_{1}-m_{2}} d_{2}^{\prime \prime}, \ldots, p^{m_{1}-m_{t}} d_{t}^{\prime \prime}, p^{m_{1}}\right)= \\
=\text { НОД }\left(d_{1}, p^{m_{1}-m_{2}} d_{2}, \ldots, p^{m_{1}-m_{t}} d_{t}, p^{m_{1}}\right), \\
\text { НОД }\left(d_{1}^{\prime \prime}, d_{2}^{\prime \prime}, p^{m_{2}-m_{3}} d_{3}^{\prime \prime}, \ldots, p^{m_{2}-m_{t}} d_{t}^{\prime \prime}, p^{m_{2}}\right)= \\
=\text { НОД }\left(d_{1}, d_{2}, p^{m_{2}-m_{3}} d_{3}, \ldots, p^{m_{2}-m_{t}} d_{t}, p^{m_{2}}\right), \\
\ldots \\
\text { НОД }\left(d_{1}^{\prime \prime}, \ldots, d_{t-1}^{\prime \prime}, p^{m_{t-1}-m_{t}} d_{t}^{\prime \prime}, p^{m_{t-1}}\right)= \\
=\text { НОД }\left(d_{1}, \ldots, d_{t-1}, p^{m_{t-1}-m_{t}} d_{t}, p^{m_{t-1}}\right), \\
\text { НОД }\left(d_{1}^{\prime \prime}, \ldots, d_{t-1}^{\prime \prime}, d_{t}^{\prime \prime}, p^{m_{t}}\right)=\text { НОД }\left(d_{1}, \ldots, d_{t-1}, d_{t}, p^{m_{t}}\right),
\end{array}\right.
$$

где $d_{j}^{\prime \prime}=$ НОД $\left((n-1) u_{1 j}+l_{1 j}^{\prime}, \ldots,(n-1) u_{r_{j} j}+l_{r_{j} j}^{\prime}\right)$ для всех $j=1, \ldots, t$.

Осталось показать, что из справедливости системы (52) следует выполнение равенств системы (8). С помощью произвольно выбранного $j$-го равенства

$$
\begin{aligned}
& q_{j}=\operatorname{HОД}\left(d_{1}^{\prime \prime}, \ldots, d_{j}^{\prime \prime}, p^{m_{j}-m_{j+1}} d_{j+1}^{\prime \prime}, \ldots, p^{m_{j}-m_{t}} d_{t}^{\prime \prime}, p^{m_{j}}\right)= \\
& =\operatorname{HОД}\left(d_{1}, \ldots, d_{j}, p^{m_{j}-m_{j+1}} d_{j+1}, \ldots, p^{m_{j}-m_{t}} d_{t}, p^{m_{j}}\right)
\end{aligned}
$$


системы (52) покажем справедливость $j$-го равенства

$$
\begin{aligned}
& q_{j}^{\prime}=\operatorname{HОД}\left(d_{1}^{\prime}, \ldots, d_{j}^{\prime}, p^{m_{j}-m_{j+1}} d_{j+1}^{\prime}, \ldots, p^{m_{j}-m_{t}} d_{t}^{\prime}, p^{m_{j}}, n-1\right)= \\
& =\operatorname{HОД}\left(d_{1}, \ldots, d_{j}, p^{m_{j}-m_{j+1}} d_{j+1}, \ldots, p^{m_{j}-m_{t}} d_{t}, p^{m_{j}}, n-1\right)=q_{j}^{\prime \prime}
\end{aligned}
$$

системы (8). Так как $q_{j}^{\prime}$ делит каждое число, стоящее в левой части равенства (54), то $q_{j}^{\prime}$ будет делить каждое число, стоящее в левой части равенства (53), а значит, $q_{j}^{\prime}$ делит $q_{j}$, откуда следует делимость каждого числа, стоящего в правой части равенства (54), на $q_{j}^{\prime}$, тогда и $q_{j}^{\prime \prime}$ делится на $q_{j}^{\prime}$. Аналогично доказывается делимость $q_{j}^{\prime}$ на $q_{j}^{\prime \prime}$. Значит, $q_{j}^{\prime}=q_{j}^{\prime \prime}$. Проводя такие рассуждения для каждого $j=1, \ldots, t$, мы тем самым покажем, что из справедливости системы (52) следует выполнение системы равенств (8). Теорема доказана.

Порядки $p^{m_{j}}$ абелевых полуциклических прямых множителей и определяющий набор наибольших общих делителей $D_{1}, \ldots, D_{t}$ из разложения (4) назовем инвариантами абелевой $n$-арной группы $\langle G, f\rangle$ из теоремы 4

Теорема 5. Своими инвариантами абелева п-арная р-группа определяется с точностью до изоморфизма

Доказательство. Пусть две абелевы $n$-арные группы $\langle G, f\rangle$ и $\left\langle G^{\prime}, f^{\prime}\right\rangle$ имеют одинаковые инварианты, пусть $\langle G, f\rangle$ изоморфна разложению (4), а $\left\langle G^{\prime}, f^{\prime}\right\rangle$ изоморфна разложению (7). Рассмотрим еще одно прямое произведение

$$
\prod_{i=1}^{r_{1}} a b l_{l_{i 1}^{\prime} a_{i 1}}\left(a_{i 1}\right) \times \prod_{i=1}^{r_{2}} a b l_{l_{i 2}^{\prime} a_{i 2}}\left(a_{i 2}\right) \times \ldots \times \prod_{i=1}^{r_{t}} a b l_{l_{i t}^{\prime} a_{i t}}\left(a_{i t}\right) .
$$

Заметим, что $n$-арная группа (4) равна $a b l_{d} A$, где $A=\sum_{j=1}^{t} \sum_{i=1}^{r_{j}}\left(a_{i j}\right), d=$ $\sum_{j=1}^{t} \sum_{i=1}^{r_{j}} l_{i j} a_{i j}$, а $n$-арная группа (55) равна $a b l_{d^{\prime}} A$, где $d^{\prime}=\sum_{j=1}^{t} \sum_{i=1}^{r_{j}} l_{i j}^{\prime} a_{i j}$. В силу Леммы 1 из [9] можно считать, что все $l_{i j}$ и $l_{i j}^{\prime}$ удовлетворяют условиям: $0 \leqslant l_{i j}, l_{i j}^{\prime}<$ НОД $\left(n-1, p^{m_{j}}\right)$ и $l_{i j}, l_{i j}^{\prime}$ делят НОД $\left(n-1, p^{m_{j}}\right)$. Так как определяющие наборы наибольших общих делителей для разложений (4) и (55) одинаковы, то, согласно лемме 1 , найдется такой автоморфизм $\sigma$ группы $A$, что $\sigma(d)=d^{\prime}$, а значит, по теореме 1 имеем изоморфизм $n$-арных групп (4) и (55). Изоморфизм (55) и (7) очевиден. Теорема доказана.

Пусть $m$ - натуральное число. Запишем его в виде суммы

$$
m=\sum_{j=1}^{t} \sum_{i=1}^{r_{j}} m_{j i}, \quad m_{11}=\ldots=m_{1 r_{1}}>m_{21}=\ldots=m_{2 r_{2}}>\ldots>m_{t 1}=\ldots=m_{t r_{t}} \geqslant 1 .
$$

Число всех таких разбиений с $r=1,2, \ldots$, где $r=r_{1}+r_{2}+\ldots+r_{t}$ и $t-$ количество различных слагаемых в разбиении $m$, обозначим через $p(m)$. Для каждого разбиения $\delta_{s}(m)$ вида $(56)(s=1, \ldots, p(m))$ определим число $q(s, m)$, равное количеству всевозможных наборов наибольших общих делителей вида (5), где $m_{j}=m_{i j}$ для $j=1, \ldots, t$ и $i=1, \ldots, r_{j}$. Например, для $m=4$ имеем $p(4)=5$ разбиений:

$\delta_{1}(4): 4=1+1+1+1, \delta_{2}(4): 4=2+1+1, \delta_{3}(4): 4=2+2, \delta_{4}(4): 4=3+1, \delta_{5}(4): 4=4$.

Для каждого из указанных разбиений при $n=3$ и $p=2$ определяем количество всевозможных наборов вида $(5): q(1,4)=2, q(2,4)=3, q(3,4)=2, q(4,4)=3, q(5,4)=$ 2 . 
Следствие 1. Число неизоморфных абелевых $n$-арных групп порядка $p^{m}$ равно

$$
\sum_{s=1}^{p(m)} q(s, m)
$$

Например, число неизоморфных абелевых тернарных групп порядка 16 равно 12, хотя число неизоморфных обычных (бинарных) абелевых групп того же порядка 16 равно 5 . Если циклическую группу порядка $k$ обозначить $Z_{k}$, то упомянутые выше неизоморфные абелевы тернарные группы порядка 16 имеют вид:

$$
\begin{gathered}
a b l_{1} Z_{2} \times a b l_{1} Z_{2} \times a b l_{1} Z_{2} \times a b l_{1} Z_{2}, \quad a b l_{0} Z_{2} \times a b l_{0} Z_{2} \times a b l_{0} Z_{2} \times a b l_{0} Z_{2}, \\
a b l_{1} Z_{4} \times a b l_{1} Z_{2} \times a b l_{1} Z_{2}, \quad a b l_{0} Z_{4} \times a b l_{1} Z_{2} \times a b l_{1} Z_{2}, \quad a b l_{0} Z_{4} \times a b l_{0} Z_{2} \times a b l_{0} Z_{2}, \\
a b l_{1} Z_{4} \times a b l_{1} Z_{4}, \quad a b l_{0} Z_{4} \times a b l_{0} Z_{4}, \quad a b l_{1} Z_{8} \times a b l_{1} Z_{2}, \quad a b l_{0} Z_{8} \times a b l_{1} Z_{2}, \\
a b l_{0} Z_{8} \times a b l_{0} Z_{2}, \quad a b l_{1} Z_{16}, \quad a b l_{0} Z_{16} .
\end{gathered}
$$

Если $n-1$ не делится на простое число $p$, то разложение конечной абелевой $n$-арной $p$-группы в прямое произведение абелевых полуциклических $n$-арных $p$-групп значительно упрощается. Дело в том, что в этом случае имеем только один определяющий набор наибольших общих делителей (5): все $D_{j}$ равны 1 . Кроме того, любая конечная абелева полуциклическая $n$-арная $p$-группа изоморфна циклической $n$-арной группе (порожденной одним элементом) того же порядка (см. Следствие 1 , [7]). А значит, верно (как и для групп)

Циклическая $n$-арная группа является $n$-арным аналогом обычной (бинарной) циклической группы. Она определяется следующим образом: вначале результат $k$-кратного применения операции $f$ в $n$-арной группе к $k(n-1)+1$ одинаковым элементам, равным элементу $a$, назовем неотрицательной $k$-й $n$-арной степенью элемента $a$ и обозначим $a^{\langle k\rangle}$. Положим $a^{\langle 0\rangle}=a$. Отрицательную $k$-ю $n$-арную степень элемента $a$ определим как решение уравнения $f(\underbrace{a, \ldots, a}_{-k(n-1)}, x)=a$. Таким образом, при $k \geqslant 0$ верно равенство $a^{\langle k\rangle}=f(\underbrace{a, \ldots, a}_{k(n-1)+1}, x)$, а при $k<0$ верно равенство $f(\underbrace{a, \ldots, a}_{-k(n-1)}, a^{\langle k\rangle})=a$. В $n$-арной группе для фиксированного элемента $a$ множество $\langle a\rangle$ всех $n$-арных степеней элемента $a$ является $n$-арной подгруппой (которую называют циклической), порожденной элементом $a$.

Следующее утверждение аналогично соответствующей теореме из теории групп.

Следствие 2. (Теорема 9, [4]) Если р не делит $n-1$, то каждая конечная абелева $n$-арная р-группа изоморфна прямому произведению ииклических $n$-арных $p$-групп. Если существуют два таких изоморфизма

$$
\langle G, f\rangle \cong a b l_{l_{1} a_{1}}\left(a_{1}\right) \times \ldots \times a b l_{l_{r} a_{r}}\left(a_{r}\right) \cong a b l_{l_{1}^{\prime} b_{1}}\left(b_{1}\right) \times \ldots \times a b l_{l_{s}^{\prime} b_{s}}\left(b_{s}\right),
$$

mо $r=s$ u $a b l_{l_{i} a_{i}}\left(a_{i}\right) \cong a b l_{l_{j}^{\prime} b_{j}}\left(b_{j}\right)$ при некотором упорядочении последних. 


\section{3. Основная теорема о конечных абелевых $n$-арных группах.}

Опираясь на разложение конечной абелевой $n$-арной группы в прямое произведение примарных абелевых $n$-арных групп (теорема 2) и на его единственность, а также на теоремы 3 и 4, мы непосредственно приходим к следующему основному утверждению о конечных абелевых $n$-арных группах.

Теорема 6. Всякая конечная абелева $n$-арная группа изоморфна прямому призведению примарных абелевых полуциклических п-арных групп. Любые два таких разложения имеют одинаковые числа множителей каждого порядка, и по каждому простому делителю порядка этой п-арной группы произведения примарных множителей в этих разложениях имеют одинаковые определяющие наборы наибольших общих делителей.

Следствие 3 (Теорема 11, [4]). Всякая конечная абелева n-арная группа, порядок которой взаимно прост с $n-1$, изоморфна прямому произведению примарных ицклических п-арных групп. Любые два таких изоморфизма в этом случае имеют одинаковые числа множителей каждого порядка.

Заимствуя терминологию из теории групп, мы, как и в случае примарных $n$-арных групп, назовем инвариантами абелевой $n$-арной групnъ $\langle G, f\rangle$ порядки $p_{s}^{m_{s j}}$ примарных абелевых полуциклических множителей в разложении абелевой $n$-арной группы $\langle G, f\rangle$ из теоремы 6 вместе с определяющими наборами наибольших общих делителей $D_{s 1}, \ldots, D_{s t_{s}}$ по каждому простому делителю $p_{s}$ порядка $|G|$. Выпишем все инварианты, расположив их в строки, которые отвечают различным простым делителям порядка $|G|$ :

$$
\begin{gathered}
\underbrace{p_{1}^{m_{11}}, \ldots, p_{1}^{m_{11}}}_{r_{11}}, \ldots, \underbrace{p_{1}^{m_{1 t_{1}}}, \ldots, p_{1}^{m_{1 t_{1}}}}_{r_{1 t_{1}}}, D_{11}, \ldots, D_{1 t_{1}} ; m_{11}>\ldots>m_{1 t_{1}} ; \\
\underbrace{p_{k}^{m_{k 1}}, \ldots, p_{k}^{m_{k 1}}}_{r_{k 1}}, \ldots, \underbrace{p_{k}^{m_{k t_{k}}}, \ldots, p_{k}^{m_{k t}}}_{r_{k t_{k}}}, D_{k 1}, \ldots, D_{k t_{k}} ; m_{k 1}>\ldots>m_{k t_{k}} ;
\end{gathered}
$$

Для фиксированного простого делителя $p_{s}$ порядка абелевой $n$-арной группы $\langle G, f\rangle$ рассмотрим произведение примарных множителей из разложения $\langle G, f\rangle$ :

$$
\prod_{i=1}^{r_{s 1}} a b l_{l_{i 1} a_{s 1}}\left(a_{s 1}\right) \times \ldots \times \prod_{i=1}^{r_{s t_{s}}} a b l_{l_{i t_{s}} a_{s t_{s}}}\left(a_{s t_{s}}\right)
$$

где для $j=1, \ldots, t_{s}$ имеем $\left|a_{s j}\right|=p_{s}^{m_{s j}}$. Произведение (57) имеет определяющий набор наибольших общих делителей

$$
\left\{\begin{array}{l}
D_{s 1}=\mathrm{HОД}\left(d_{s 1}, p^{m_{s 1}-m_{s 2}} d_{s 2}, \ldots, p^{m_{s 1}-m_{s t_{s}}} d_{s t_{s}}, p^{m_{s 1}}, n-1\right), \\
D_{s 2}=\mathrm{HОД}\left(d_{s 1}, d_{s 2}, p^{m_{s 2}-m_{s 3}} d_{s 3}, \ldots, p^{m_{s 2}-m_{s t_{s}}} d_{s t_{s}}, p^{m_{s 2}}, n-1\right), \\
\cdots \\
D_{s t_{s}-1}=\mathrm{HОД}\left(d_{s 1}, \ldots, d_{s t_{s}-1}, p^{m_{s t_{s}-1}-m_{s t_{s}}} d_{s t_{s}}, p^{m_{s t_{s}-1}}, n-1\right), \\
D_{s t_{s}}=\mathrm{HOД}\left(d_{s 1}, \ldots, d_{s t_{s}-1}, d_{s t_{s}}, p^{m_{t_{s}}}, n-1\right),
\end{array}\right.
$$

где $d_{s j}=$ НОД $\left(l_{1 j}, \ldots, l_{r_{s j} j}\right)$, для всех $j=1, \ldots, t_{s}$. 
Среди всех изоморфных копий произведения (57) удобнее всего рассматривать произведение

$$
a b l_{d_{s 1} a_{s 1}}\left(a_{s 1}\right) \times \prod_{i=2}^{r_{s 1}} a b l_{0}\left(a_{s 1}\right) \times \ldots \times a b l_{d_{s t_{s}} a_{s t_{s}}}\left(a_{s t_{s}}\right) \times \prod_{i=2}^{r_{s t_{s}}} a b l_{0}\left(a_{s t_{s}}\right) .
$$

Так как $\prod_{i=2}^{r_{s 1}} a b l_{0}\left(a_{s 1}\right) \times \ldots \times \prod_{i=2}^{r_{s t_{s}}} a b l_{0}\left(a_{s t_{s}}\right) \cong a b l_{0} \prod_{j=1}^{t_{s}} \prod_{i=2}^{r_{s j}}\left(a_{s j}\right)$ (см. [10], стр.157), то произведение (59) изоморфно произведению

$$
\prod_{j=1}^{t_{s}} a b l_{d_{s j} a_{s j}}\left(a_{s j}\right) \times a b l_{0} \prod_{j=1}^{t_{s}} \prod_{i=2}^{r_{s j}}\left(a_{s j}\right) .
$$

Тогда разложение $\langle G, f\rangle$ будет иметь вид

$\langle G, f\rangle \cong \prod_{j=1}^{t_{1}} a b l_{d_{1 j} a_{1 j}}\left(a_{1 j}\right) \times a b l_{0} \prod_{j=1}^{t_{1}} \prod_{i=2}^{r_{1 j}}\left(a_{1 j}\right) \times \ldots \times \prod_{j=1}^{t_{k}} a b l_{d_{k j} a_{k j}}\left(a_{k j}\right) \times a b l_{0} \prod_{j=1}^{t_{k}} \prod_{i=2}^{r_{k j}}\left(a_{k j}\right)$,

или, еще проще,

$$
\langle G, f\rangle \cong \prod_{s=1}^{k} \prod_{j=1}^{t_{s}} a b l_{d_{s j} a_{s j}}\left(a_{s j}\right) \times a b l_{0} \prod_{s=1}^{k} \prod_{j=1}^{t_{s}} \prod_{i=2}^{r_{s j}}\left(a_{s j}\right) .
$$

В качестве примера перечислим все абелевы тернарные группы порядка 36:

\begin{tabular}{|c|c|}
\hline Абелевы тернарные группы порядка 36 & Инварианты \\
\hline$a b l_{0} Z_{4} \times a b l_{0} Z_{9} \cong a b l_{0}\left(Z_{4} \times Z_{9}\right)$ & 4,$2 ; 9,1$ \\
\hline$a b l_{1} Z_{4} \times a b l_{0} Z_{9}$ & 4,$1 ; 9,1$ \\
\hline$a b l_{0} Z_{2} \times a b l_{0} Z_{2} \times a b l_{0} Z_{9} \cong a b l_{0}\left(Z_{2} \times Z_{2} \times Z_{9}\right)$ & $2,2,2 ; 9,1$ \\
\hline$a b l_{1} Z_{2} \times a b l_{0} Z_{2} \times a b l_{0} Z_{9} \cong a b l_{1} Z_{2} \times a b l_{0}\left(Z_{2} \times Z_{9}\right)$ & $2,2,1 ; 9,1$ \\
\hline$a b l_{1} Z_{4} \times a b l_{0} Z_{3} \times a b l_{0} Z_{3} \cong a b l_{1} Z_{4} \times a b l_{0}\left(Z_{3} \times Z_{3}\right)$ & 4,$1 ; 3.3,1$ \\
\hline$a b l_{0} Z_{4} \times a b l_{0} Z_{3} \times a b l_{0} Z_{3} \cong a b l_{0}\left(Z_{4} \times Z_{3} \times Z_{3}\right)$ & 4,$2 ; 3,3,1$ \\
\hline$a b l_{1} Z_{2} \times a b l_{0} Z_{2} \times a b l_{0} Z_{3} \times a b l_{0} Z_{3} \cong a b l_{1} Z_{2} \times a b l_{0}\left(Z_{2} \times Z_{3} \times Z_{3}\right)$ & $2,2,1 ; 3.3,1$ \\
\hline$a b l_{0} Z_{2} \times a b l_{0} Z_{2} \times a b l_{0} Z_{3} \times a b l_{0} Z_{3} \cong a b l_{0}\left(Z_{2} \times Z_{2} \times Z_{3} \times Z_{3}\right)$ & $2,2,2 ; 3,3,1$ \\
\hline
\end{tabular}

\section{Список литературы}

1. Курош А. Г., Общая алгебра. Лекиии 1969-1970 уч. года, Наука, Москва, 1974.

2. Timm J., Kommutative n-Gruppen, Diss, Hamburg, 1967.

3. Dudek W. A., Michalski J., "On retracts of polyadic groups", Demonstr. Math., 17 (1984), 281-301.

4. Бощенко А. П., Щучкин Н. А., "Конечные абелевы $n$-арные группы", Чебышевский сборник, XII:2(38) (2011), 5-14.

5. Гальмак А. М., "Полуабелевы $n$-арные группы с идемпотентами", Веснік ВДУ им. П. М. Машэрава, 2:12 (1999), 56-60.

6. Dudek W. A., "Remarks to Glazek's results on n-ary groups", Discuss. Math., General Algebra and Appl., № 27, 2007, 199-233.

7. Щучкин Н. А., "Полуциклические $n$-арные группы”, Известия ГГУ им. Ф. Скорины, 3:54 (2009), 186-194. 
8. Курош А. Г., Теория групn, 3-е изд., Наука, Москва, 1967.

9. Glazek K., Michalski J., Sierocki A I., "On evaluation of numbers of some polyadic groups", 1985, 157-171.

10. Гальмак А. М., n-арные группы. Часть I, Гомельский гос. ун-т им. Ф. Скорины, Гомель, 2003.

Статья поступила 06.12.2013. 